\title{
Supersymmetric Dark Matter
}

\author{
Csaba Balázs and Daniel Carter \\ Centre of Excellence for Particle Physics at the Terascale and \\ Monash Centre for Astrophysics \\ School of Physics, Monash University \\ Australia
}

\section{Introduction}

The standard model of particle physics excellently describes all known fundamental particles and forces (with the exception of gravity). Over the last several decades, this theory has become established through a preponderance of experimental data, culminating in the detection of the top quark at the Tevatron collider. However, while there has never been a direct observation of a particle that does not fit in the standard model, there are compelling reasons to believe that such particles exist, and so the standard model is not a complete description of Nature.

The most noteworthy piece of evidence in this case comes from astrophysics: the enigmatic dark matter. Dark matter was postulated to explain why stars in galaxies appeared to orbit faster than the gravitational attraction of the luminous mass would imply. This hypothesis, that there is more matter in the universe than is luminous, has been confirmed by observations in galactic structure formation, gravitational lensing, and most importantly in the cosmic microwave background radiation. Relics from the early universe, small deviations from uniformity in the cosmic microwave background radiation provide details on the matter content of the universe, and in particular it places stringent upper bounds on the amount of baryonic matter, ruling out atomic matter as an explanation for dark matter. Ultimately, it has been determined that any potential particle of dark matter must satisfy several requirements. It must be massive, apart from gravitation it may only interact very weakly, it must be stable on a cosmic time scale, and it must be non-relativistic (having average temperature much below its mass scale). This immediately excludes any particle in the standard model.

Other possible shortcomings with the standard model are also emerging. For example, in certain precision experiments, such as the measurement of the anomalous magnetic moment of the muon, discrepancies seem to be appearing between the experimentally measured value and the value predicted by the standard model. These results may indicate the presence of additional contributions from currently unknown particles.

We are therefore led to consider new theories that extend the standard one. Any such theory should explain these new phenomena, but must also reproduce the myriad successes of the standard model. One of the most promising ideas is the extension of the standard model by the introduction of supersymmetry. Supersymmetry is the unification of fermions and bosons, in other words the unification of matter and force. Under supersymmetry, all fermions and bosons are considered as components of a superfield, and hence for every fermionic field in a 
supersymmetric theory there will be a corresponding bosonic field (and vice versa) with the same mass and quantum numbers. If we impose supersymmetry on the standard model, we therefore necessarily introduce a number of new particles: a new spin zero field for both spin degrees of freedom for each quark and lepton, a new spin-half field for each of the force carrier bosons, and additional spin-half fields for the complex degrees of freedoms of the Higgs bosons. This gives us the Minimal Supersymmetric Standard Model (MSSM). From the new electrically neutral fermionic fields we construct the neutralinos, the lightest of which satisfies all the basic requirements for being a dark matter particle. As well as this, supersymmetry neutralises the quadratic divergences in the quantum corrections to the Higgs mass, solving the so-called hierarchy problem.

Of course, supersymmetry is not preserved in the universe today, or we would certainly have detected the presence of at least some super-particles. We know supersymmetry must be broken. However the precise mechanism can so far only be speculated. One particularly interesting idea is supergravity, in which supersymmetry is elevated to a local symmetry and as a result is broken via gravity. Our investigations concern the Next to Minimal Supersymmetric Standard Model (NMSSM) in which the MSSM is augmented by a gauge singlet superfield subject to discrete symmetries, which alleviates naturalness issues of the Higgs mass parameter. On this scenario we impose supergravity constraints, creating the NmSuGra model.

Unsurprisingly though, testing a new theory for viability can pose a considerable challenge. Not only is it highly complex to calculate predictions for a given model, but invariably there will be degrees of freedom in the model which are not constrained by theoretical considerations, which must therefore become free parameters. Varying these creates a multiplicity of possibilities that can easily become unmanageable, and so sophisticated statistical techniques are required to keep track of supersymmetric predictions. We adopt Bayesian probability theory as the appropriate general formalism for examining the plausibility of a model, and our results employ sophisticated numerical algorithms to explore the parameter space of the theory. For each set of parameters, predictions are calculated for a comprehensive selection of pertinent observables, including dark matter abundance, collider limits and precision measurements, which are used to assign a likelihood value.

While there have not so far been any direct observations of dark matter or of supersymmetry, there are good prospects for such events to occur in the near future. The CERN Large Hadron Collider (LHC) will have the capacity to create supersymmetric particles if they are light enough, and may produce detectable signals in the form of missing energy from escaped neutralinos. Furthermore, experiments exist with the aim of detecting cosmic dark matter directly through nuclear scattering events in underground vats. We find that the chances of detection for NmSuGra (and a broad class of similar theories) at these experiments are quite favourable.

\section{Dark matter}

The existence of dark matter is one of the most significant enduring mysteries of modern particle astrophysics, every bit as enigmatic as its name suggests. Dark matter is the generic name for an unknown substance which is possibly made of more than one constituents. It has never been directly observed, but it is now believed to constitute the bulk of matter existing in the Universe today. 
Experimental evidence for the existence of dark matter is overwhelming. Historically, dark matter was proposed in order to explain a number of anomalous astrophysical observations in which the effects of gravity could not be accounted for by the measured mass of luminous matter. Initial evidence for dark matter came from the curious observation that outer parts of galaxies rotate faster than they should. In the seminal observations of Vera Rubin, the orbital velocities of stars were measured as a function of distance from the center of the Andromeda Galaxy (Rubin \& Ford, 1970). Rubin and collaborators determined that the velocities did not vary greatly with distance, whereas the centralised distribution of visible mass would be consistent with velocities decreasing rapidly as radial distance increases. This discrepancy could be resolved by the presence of a galactic halo of some non-visible, gravitating matter, that has subsequently come to be known as dark matter.

Numerous other observations, originating from vastly different length scales, support the dark matter hypothesis. Light bending around the enormous gravitational potential of Galaxy super-clusters, an effect coined as gravitational lensing, indicates significantly more mass within these clusters that meets the eye (Kneib et al., 1996). Simulations of Galaxy and larger structure formation have determined that the clustering of galaxies observed in the Universe today could not occur under the gravitational effects of the matter that can be seen (Abazajian et al., 2009). An interesting special case, the Bullet Cluster, is comprised of two galactic clusters undergoing a violent, cosmic collision. Individual galaxies and intergalactic gas, consisting of charged cosmic rays, are visible (the latter mainly due to its $\mathrm{X}$-ray emissions). Via gravitational lensing it was observed that the concentration of mass was separated from the visible matter in the Bullet Cluster. In fact, the bulk of mass was carried by an invisible, non-luminous agent (Markevitch et al., 2004). A simple explanation of these observations is that visible matter, which interacts more strongly than dark matter, was left behind closer to the core of the collision while dark matter halos surrounding them, interacting weaker, rushed forward and the two became separated from each other (Clowe et al., 2006).

The most precise value for the amount of dark matter in the Universe comes from measurements of anisotropies in the cosmic microwave background, the CMB. In the early Universe, after quarks bound into atomic nuclei, matter existed in an opaque plasma state. As the Universe cooled and neutral atoms were formed, the thermal radiation produced by this plasma was able to propagate freely. Today we can measure the remnant of this radiation as a highly uniform microwave signal observable from all directions of the sky. The small deviations from uniformity, density fluctuations in the early plasma, are the cosmic seeds of structure formation. The amount of the non-uniformity depends on the amount of matter in the early Universe. This is because more matter gravitates stronger thus, collapsing under the effect of stronger gravity, more matter creates higher density fluctuations. Thus the power spectrum of the CMB can be used to deduce the matter composition of the Universe (Komatsu et al., 2011).

The CMB spectrum also gives us the density of ordinary atomic matter built of baryons, which are bound states of quarks. From the $\mathrm{CMB}$ we can deduce that only about twenty percent of the total matter density is baryonic. This is confirmed, with excellent precision, by the observation of the abundances of the lightest elements in the Universe (Amsler et al., 2008). The fact that baryonic energy density is only a fraction of the total matter density excludes the simplest possibility that dark matter is composed of regular atomic matter.

But then what is dark matter made of? To address this question we have to ask: What do we know about the properties of dark matter so far? From astrophysical measurements 
we deduced that dark matter is non-baryonic. It must also be stable against decay, at least on the timescale of the lifetime of the Universe, otherwise it could not change gravitational observations today. Structure formation further indicates that dark matter is cold, which means that the speed of the thermal motion of dark matter constituents at the present average temperature of the Universe is much lower than the speed of light. Finally, from observations such as the Bullet Cluster we know that dark matter must be very weakly interacting. It may only interact via gravity or a similarly feeble force.

The above properties exclude the possibility that the bulk of dark matter is made of known stable particles such as protons, electrons or neutrinos. Thus, there are numerous competing theories and speculations regarding what dark matter might be made of. It appears that one of the simplest avenues toward solving the dark matter puzzle is to postulate the existence of an undiscovered, exotic particle. The density evolution of such dark matter particles over the eons can be traced by thermodynamical calculations (Kolb \& Turner, 1990). These calculations show that dark matter density decreased over the history of the Universe due to expansion of space and annihilation between dark matter particles and anti-particles. At some temperature in the early Universe, known as the freeze-out temperature, the density of dark matter decreased to the point that annihilation became negligible to the further evolution of the density, which is now dominated by expansion. What remains today is called the relic abundance, or relic density, of dark matter. The present dark matter abundance depends strongly on the specific properties of the dark matter particle, and is thus essential for discriminating between different theories of dark matter.

Another important question is: How can we observe dark matter particles? Three main avenues were suggested so far: indirect, direct and collider detection. Indirect detection of dark matter utilizes the fact that annihilation to standard particles continues, particularly in localised high-density regions that re-collapsed under the effects of gravity. As today dark matter particles are cold, their non-relativistic thermal kinetic energy is negligible compared to their mass. Thus, the annihilation energy is dominated by the mass of the dark matter particle. Hence it is anticipated that dark matter annihilation will produce standard particle pairs at energies equaling the mass of the dark matter particle. The hope is to detect such a dramatic, peaked signal on the background of the relatively smooth cosmic ray flux of standard astrophysical origin.

Direct dark matter detection experiments aim to find traces of dark matter in ground based detectors. In these highly shielded detectors dark matter particles may bounce into atomic nuclei, which is typically a pure noble gas or a crystalline metal. The boosted nuclei in turn dissipate their energy in the form of heat, light or ionization. Extremely sensitive phonon, scintillation and ionization detectors then can observe this signal of dark matter-nucleon collision.

The ultimate method of discovering dark matter particles is producing them in the laboratory. If dark matter particles are heavy, relativity dictates that this requires colliding standard particles at large energies. If dark matter interactions are very weak, then due to the probabilistic nature of quantum physics, the process also necessitates many of these collisions. The highest energy and luminosity particle collider presently is the Large Hadron Collider at CERN. So all eyes fall on the LHC when it comes to making dark matter on Earth. Eventually, answering the question of what dark matter actually is requires the synthesis of particle physics with astrophysics, the understanding of phenomena at the largest and the smallest scales in the same time. 


\section{Supersymmetry}

Over the second half of the twentieth century, the standard model of particle physics became established as the theoretical framework describing everything that was known about particle physics. With the exception of gravity the standard model incorporates all the fundamental forces that have ever been observed: the electromagnetic, weak and strong forces. These forces are physical consequences of the gauge symmetries of the standard model. Corresponding to these symmetries are three types of force carriers: the photon, weak bosons and gluons. Matter in the standard model is represented by fermions: six quarks and six leptons which are arranged into three generations. An essential, albeit curious, feature of the standard model is that weak interactions violate chirality: electrically charged weak bosons only mediate forces between left-handed fermions. Due to this the standard model exhibits a chiral symmetry, that is the left- and right-handed fermions transform independently. All these features and properties of the standard model have been repeatedly confirmed in experiment, some with a remarkable precision.

However, in recent years data has been accumulating for which the standard model is insufficient to explain. The existence of dark matter is one of the most compelling evidences. A dark matter particle must be electrically neutral and it cannot be baryonic. It must also be stable and have sufficient mass. This leaves no viable candidate for dark matter in the standard model. There are other experimental indications that the standard model may no longer be sufficient, some of which will be discussed in section 4 .

Perhaps even more importantly there are conceptual issues the resolution of which lies outside the scope of the standard model. These include the origin of fundamental particle properties such as mass, spin and their various charges such as electromagnetic, weak and strong charges. A further puzzle is the number and variety of elementary particles: six types of quarks and leptons, and three types of force carriers.

The most immediate conceptual problem of the standard model is the underlying dynamics of electroweak symmetry breaking. Electroweak symmetry breaking generates the masses of all elementary particles and the necessary agent of this is the Higgs boson. The Higgs boson, so far a hypothetical particle, is one of a kind - the only spin zero fundamental particle. Such scalar particles spell trouble in the standard model. The standard gauge and chiral structure protects the masses of matter particles and the force carriers. In any quantum field theory, such as the standard model, particle masses receive higher order corrections from vacuum fluctuations. These corrections can grow essentially unbounded unless there is a mechanism to limit them. There is no such mechanism for the Higgs boson mass in place in the standard model. Unless something protects the Higgs mass, electroweak symmetry breaking collapses in the standard model. This is called the hierarchy problem because the unbounded growth of the Higgs mass is thought to be due to the large difference between the weak and Planck energy scales.

It turns out that it is possible to utilize the existing structure of the standard model to extend the protection to the Higgs mass. There would be no hierarchy problem if either the existing gauge or chiral symmetries of the standard model could be used to protect the Higgs mass. This is only wishful thinking unless the Higgs mass is directly connected to one of these symmetries. The introduction of a new overarching symmetry, supersymmetry, does just this (Baer \& Tata, 2006). Supersymmetry demands that the Higgs boson is partnered with a chiral fermion, called the Higgsino. The Higgs and Higgsino masses have to be the same due to supersymmetry. But since the Higgsino is a chiral fermion its mass is protected by the 
standard chiral symmetry. Thus the introduction of supersymmetry extends the protection to the Higgs mass.

Supersymmetry is attractive for various other reasons. It is the only possible extension of the Poincare symmetry of space-time that is consistent with quantum field theory. Formally, we can define an operator $Q$ that acts on a fermionic state and transforms it to a bosonic state, and vice versa. It follows that $Q$ must therefore be a spin-half operator, obeying anti-commutation relations. It can be shown that the generators of the Poincare symmetry accompanied by the generators of the supersymmetry transformations extend the Poincaré group to a super-structure. Amongst other things this implies that a specific combination of supersymmetric transformations is simply a space-time shift. Such extensions of the Poincaré group are in general forbidden by the Coleman-Mandula theorem, however this theorem does not cover non-commuting operators like $Q$ (Coleman \& Mandula, 1967). According to the more general Haag-Lopuszanski-Sohnius theorem, supersymmetry is the unique possible extension of the Poincaré algebra, which is quite intriguing (Haag et al., 1975).

Supersymmetry posits that there exists a fundamental symmetry between fermionic and bosonic fields, that in fact these are merely components of a more fundamental entity called a superfield. There are two principle kinds of superfields: chiral and vector superfields. The former contain two physical scalar fields and one physical spin-half field, and are used to represent the standard model fermion fields and any scalar field such as the Higgs boson. The latter embody a (massless) vector boson and a spin-half field, and represent the standard model vector fields (before particles acquire masses). To construct a realistic supersymmetric theory, we take all the fields in the standard model and elevate them to an appropriate superfield, thereby introducing a fermionic counterpart for every boson and two scalar counterparts for every fermion. This then forms the particle content of the Minimal Supersymmetric Standard Model (MSSM).

The simplest supersymmetric particle Lagrangian is the early model by Wess \& Zumino (1974). The Wess-Zumino model describes one fermionic and two bosonic fields, or in other words a single superfield. The MSSM, the simplest supersymmetric extension of the standard model, was first constructed by Dimopoulos \& Georgi (1981), and remains the most popular supersymmetric model today. The success of the MSSM is due to its versatility. The MSSM was proposed to solve the hierarchy problem but it turned out to solve various other problems of the standard model. It, for example, contains one or more viable dark matter particles. The lightest supersymmetric particle is typically long lived, massive, electrically neutral and weakly interacting: an ideal dark matter candidate (Goldberg, 1983; Pagels \& Primack, 1982). In the MSSM various particles, such as the gravitino, the lightest nautralino, the lighest sneutrino, or even the axino can be dark matter. Unification of gauge couplings also substantially improves the MSSM compared to the standard model (Amaldi et al., 1991).

In general the super-partners will mix to form distinct mass eigenstates. The mass eigenstates of the super-partners of the electrically neutral standard electroweak bosons, for example, are called neutralinos and their electrically charged counterparts are the charginos. The lightest neutralino in the MSSM is comprised of the neutral components of the two Higgs superfields (Higgsinos) and the counterparts to the weak $W^{0}$ and $B^{0}$ bosons. Spin zero super-partners are identified by the names of their spin-half counterparts with an 's' prepended, such as 'selectron' for the scalar partners of the electron. 
Super-partners are clearly distinguished from their standard counterparts by a quantum number called R-parity. The R-parity of the fields in the MSSM are defined as

$$
R=(-1)^{3(B-L)+2 S}
$$

where $B, L$ and $S$ represent the baryon number, lepton number and spin of the field, respectively. If we assume that R-parity is a conserved quantum number, then the lightest supersymmetric particle becomes stable against decay.

The form of a supersymmetric Lagrangian including interactions between chiral and vector superfields is largely fixed by the gauge and chiral symmetries of the standard model, and the Poincaré symmetry extended to supersymmetry. Matter-Higgs interactions of a supersymmetric theory are specified by the superpotential, which is a function of renormalizable combinations of left-chiral superfields. The superpotential defining the MSSM is:

$$
\left.W_{M S S M}\right|_{\mu=0}=\mu \hat{H}_{u} \cdot \hat{H}_{d}+y_{t} \hat{Q}_{L} \cdot \hat{H}_{u} \hat{T}_{R}^{c}-y_{b} \hat{Q}_{L} \cdot \hat{H}_{d} \hat{B}_{R}^{c}-y_{\tau} \hat{L}_{L} \cdot \hat{H}_{d} \hat{L}_{R}^{c}
$$

The left-handed superfields $\hat{Q}_{L}, \hat{L}_{L}$ and $\hat{H}_{u}, d$ contain the left-handed (s)quark, (s)lepton and Higgs fields respectively, and so are weak $S U(2)_{L}$ doublets, whereas the right-handed $\hat{T}_{R}$, $\hat{B}_{R}, \hat{L}_{R}$ superfields are singlets under weak transformations. Generation indices have been suppressed for the matter fields, but in general the Yukawa couplings $y_{t}$ are $3 \times 3$ matrices in generation space.

Unlike the Yukawa couplings that originate from electroweak symmetry breaking the coefficient $\mu$ of the Higgs self-interaction term is a supersymmetric parameter. In theory its natural size is not linked to the electroweak scale at all. On the other hand in the MSSM electroweak symmetry breaking will not happen unless the the size of $\mu$ is close to the electroweak scale. This issue can be addressed, however, by considering extensions of the minimal superpotential. In the so-called next-to-minimal supersymmetric standard model we introduce a gauge-singlet superfield (Fayet, 1975), augmenting the $\mu$ term in the MSSM superpotential with:

$$
W_{N M S S M}=\left.W_{M S S M}\right|_{\mu=0}+\lambda \hat{S} \hat{H}_{u} \cdot \hat{H}_{d}+\frac{\kappa}{3} \hat{S}^{3},
$$

The scalar component $S$ of the singlet superfield acquires a vacuum expectation value $s=\langle S\rangle$ after electroweak symmetry breaking, generating $\mu=\lambda s$, where $\lambda$ is an order 1 coupling. Since the size of $s$ is naturally electroweak the $\mu$ problem of the MSSM is automatically solved. As there are no gauge couplings for $\hat{S}$, the impact on phenomenology compared to the MSSM is small, although the possibility of the singlet field mixing with the scalar fields could weaken couplings to other fields, making it harder to detect.

In a supersymmetric field theory, for every fermionic degree of freedom there must exist one bosonic degree of freedom, and vice versa, with otherwise identical quantum numbers and masses. For example, in a supersymmetric quantum electrodynamics there would exist two complex spin zero counterparts to the electron for its two spin degrees of freedom. If such super-partners existed in our observed world they would have been seen in experiments well before now, so we are lead to the conclusion that supersymmetry, if it is manifested in our Universe, must be broken at some early time. 
How supersymmetry is broken can only be speculated at present. A typical assumption is that the, probably spontaneous, breaking of supersymmetry is communicated from a hidden sector to us at a very high energy scale close to the one where the strength of all fundamental forces unify. When supersymmetry breaking happens super-partners acquire masses. If no new physics occurs between the scale of supersymmetry breaking and the electroweak scale then the renormalization group equations of the MSSM allow us to to calculate the evolution of the super-partner masses and couplings between the two scales. In turn this enables us to calculate the super-partner masses and couplings at the energy scale which is probed by experiments.

Typically supersymmetry is broken such that the breaking does not ruin the protection of the Higgs, and other spin zero super-partner, masses (Dimopoulos \& Georgi, 1981). This soft breaking also decouples the origin of supersymmetry breaking from its phenomenological consequences. It is parametrized by adding the following explicit symmetry breaking to the supersymmetric Lagrangian:

$$
\mathcal{L}_{N M S S M}^{s o f t}=\left.\mathcal{L}_{M S S M}^{s o f t}\right|_{B=0}-M_{S}^{2}|\tilde{S}|^{2}-\left(\lambda A_{\lambda} \tilde{S} H_{u} \cdot H_{d}+\frac{\kappa A_{\kappa}}{3} \tilde{S}^{3}+\text { h.c. }\right) .
$$

Here

$$
\left.\mathcal{L}_{M S S M}^{\text {soft }}\right|_{B=0}=\mathcal{L}_{\text {gaugino }}^{\text {soft }}+\mathcal{L}_{\text {scalar }}^{\text {soft }}+\mathcal{L}_{\text {tri-linear }}^{\text {soft }}
$$

contains the mass terms for the twelve gauginos $(i=1 \ldots 3, a=1 \ldots 8)$

$$
\mathcal{L}_{\text {gaugino }}^{\text {soft }}=-\frac{1}{2}\left(M_{1} \tilde{\tilde{B}} \tilde{B}+M_{2} \overline{\tilde{W}}_{i} \tilde{W}_{i}+M_{3} \overline{\tilde{G}}_{a} \tilde{G}_{a}+\text { h.c }\right),
$$

the sfermions and Higgses

$$
\begin{gathered}
\mathcal{L}_{\text {scalar }}^{\text {soft }}=-\left(M_{Q}^{2}|\tilde{Q}|^{2}+M_{T_{R}}^{2}\left|\tilde{T}_{R}\right|^{2}+M_{B_{R}}^{2}\left|\tilde{B}_{R}\right|^{2}+M_{L}^{2}|\tilde{L}|^{2}+M_{L_{R}}^{2}\left|\tilde{L}_{R}\right|^{2}+\right. \\
\left.M_{H_{u}}^{2}\left|H_{u}\right|^{2}+M_{H_{d}}^{2}\left|H_{d}\right|^{2}\right),
\end{gathered}
$$

and the so called soft tri-linear terms

$$
\mathcal{L}_{\text {tri-linear }}^{\text {soft }}=-\left(y_{t} A_{t} \tilde{Q} \cdot H_{u} \tilde{T}_{R}^{c}-y_{b} A_{b} \tilde{Q} \cdot H_{d} \tilde{B}_{R}^{c}-y_{\tau} A_{\tau} \tilde{L} \cdot H_{d} \tilde{L}_{R}^{c}+\text { h.c. }\right) .
$$

The most well studied supersymmetry breaking mechanism is known as the minimal supergravity motivated (mSuGra) model, in which supersymmetry breaking is mediated via gravity and the supersymmetry breaking scale is identified by the unification scale (Chamseddine et al., 1982). The idea behind supergravity is simple and elegant: supersymmetry is promoted to a local symmetry. As supersymmetry is an extension of the Poincaré space-time symmetry, elevating supersymmetry to a local symmetry, we automatically incorporate gravity. The free parameters of minimal supergravity, introduced at the unification scale and appearing in the supersymmetry breaking Lagrangian, are as follows:

$$
P=\left\{M_{0}, M_{1 / 2}, A_{0}, \tan \beta, \lambda\right\} .
$$


Here $M_{0}$ is the unified scalar mass, $M_{1 / 2}$ is the unified gaugino mass, $A_{0}$ is a unified trilinear coupling, and $\tan \beta$ is the ratio of the vacuum expectation values of the two Higgs fields, an expedient replacement for the Higgs bilinear term $B$. For the next to minimal equivalent (NmSuGra) we add $\lambda$, the coupling constant in equation 3. Together with experimentally measured standard model input values, these parameters completely define the particle mass spectrum, interactions and mixings required to compute Feynmann diagrams, and hence derive predictions for quantities of interest.

\section{Supersymmetric predictions}

Supersymmetry is a predictive theoretical framework. Once the values of the parameters have been fixed the Lagrangian is unique and we are able to make various types of theoretical predictions. The most important of these predictions are the masses of the super-partners. This set of masses is frequently referred to as the supersymmetric spectrum and computer programs calculating it called spectrum generators. The calculation of the spectrum involves two main steps. The first is following the renormalization evolution of the supersymmetric masses from the high energy scale, which is typically the unification scale, down to the low scale, which is typically the $\mathrm{TeV}$ scale probed by modern experiments (Martin \& Vaughn, 1994). In the numerical implementation a spectrum generator solves the relevant renormalisation group equations and runs the masses down from the unification scale to the weak scale. In the second step the eigenvalues of the mass matrices have to be found by diagonalizing, leading to the supersymmetric mass eigenstates. In the process of numerically solving the renormalization group equations the couplings of these mass eigenstates are also identified.

Knowledge of the supersymmetric spectrum permits the calculation of decay rates, scattering amplitudes and related probabilities. These, in turn, allow us to calculate various observables the predicted values of which can be compared to experimental measurements. Foremost among these observables is the relic abundance, the average energy density of the lightest supersymmetric particle which typically forms part of dark matter. In supergravity motivated models the lightest supersymmetric particle is the lightest neutralino. The present energy density of these neutralinos depend on the supersymmetric spectrum and couplings. Knowing these the neutralino abundance can be evolved from high temperatures to the freeze-out temperature. This requires the calculation of neutralino self-scattering (annihilation) cross sections into standard particles and neutralino scattering (co-annihilation) cross sections on other supersymmetric particles. The size of these cross sections depend on the scattering energy and thus the average temperature of the early Universe. They enter into Boltzmann's equation, which in turn determines the neutralino relic abundance as the function of temperature. The value of this abundance after freeze-out is a very important constraint on the choice of parameters in supersymmetric models.

Based on the supersymmetric spectrum cross sections of dark matter scattering on atomic nuclei can also be calculated. In supersymmetric models dark matter scattering on nuclei is mostly mediated by Higgs boson exchange. This spin-independent process is augmented by the spin-dependent $Z$ boson and pseudo-scalar exchange. Neutralinos directly couple to Higgses (or pseudo-scalars) which couple to nuclei via virtual heavy-quark loops. Neutralino-nucleon scattering can also be mediated by scalar partners of quarks. These theoretical cross sections can be compared to measurements of experiments that attempt to 
observe dark matter directly as it passes through the Earth. Such detectors are placed deep underground in order to shield them from ordinary particles, thus minimising background. Even this way charged particles created by high energy cosmic neutrinos can reach these detectors so clever event vetoing has to be applied. Since the dark matter flux is expected to vary throughout the year, as the earth orbits the sun, an annual modulation in the signal could help identifying signals of dark matter.

Another avenue to learn about dark matter particles is indirect detection. Dark matter particles can annihilate with their own anti-particles to produce charged cosmic rays or gamma rays in the Galaxy. Such cosmic rays are expected to arrive to us from regions with high dark matter number density such as the center of our Galaxy. These cosmic rays can be detected by satellites specifically designed to measure them. Recently the Fermi-LAT and PAMELA satellites enabled us to very precisely measure the cosmic electron, positron, and gamma ray fluxes as the function of their energy. These measurements are important constraints on supersymmetric models since dark matter annihilations produce electron-positron and photon pairs at energies equaling the mass of a dark matter particle. After the cosmic rays are produced and they propagate through the inter-galactic medium they lose energy. This energy loss can be followed through solving a diffusion equation with reasonable precision. Thus the shape of the observed cosmic ray spectra may carry information to us about the mass of the dark matter particle. The amplitude of a potential dark matter annihilation signal in cosmic rays depends on the number density of the dark matter particles and their annihilation cross-section into the given cosmic ray species. While the dark matter density is relatively well predicted in the vicinity of our solar system it is quite uncertain toward the center of the Galaxy. Thus, the size of an indirect detection signal suffers from various astrophysical uncertainties. Nevertheless, they already provide useful constraints on supersymmetric models.

High energy colliders have the best chance to establish the existence of supersymmetry. Because they are highly controlled experiments they can provide us with the most comprehensive information about a given supersymmetric model. The center of mass energies of current generation colliders are sufficient to reach a significant portion of the parameter space of various supersymmetric models. The LHC, as the highest energy and luminosity collider, leads the way in the search for supersymmetry. Super-particles most likely to be produced at the LHC are the lightest squarks or the gluino, which will invariably decay in cascades. If R-parity is conserved, the final products must include the lightest supersymmetric particle, which is the lightest neutralino in mSuGra. Ironically, the lightest neutralino will not be directly detected since its interactions are so weak that it will simply fly through the LHC detectors. However, balancing energy and momentum event by event experimentalists will be able to determine its mass and even its couplings with reasonable precision. If this happens, we will be able to assess the portion of dark matter that is made of neutralinos. Even in the lack of detecting supersymmetric particles the LHC will be able to constrain supersymmetry by searching for Higgs bosons. Within the MSSM, for example, there is a strict upper limit of the mass of the lightest Higgs boson and the LHC will be able to probe this limit. If the MSSM is realized in Nature the LHC has to find a Higgs boson below this limit.

Contributions from exotic particles are expected to be important in the case of rare decays. These are decay modes which are forbidden at the lowest order in perturbation theory due to the violation of various quantum numbers, but may proceed through higher order channels mediated by virtual loops. The probability of such decays is hence quite low, however this 
means that each higher order diagram will have a greater relative impact. One example is the rate of the rare decay $b \rightarrow s \gamma$, which is considerably less than typical hadronic decay rates. Since supersymmetric contributions to $b \rightarrow s \gamma$ depend on super-partner masses and couplings, the experimental measurement can put bounds on acceptable super particle mass ranges.

There are precision low energy measurements which are sensitive to physics beyond the standard model. Via their quantum fluctuations supersymmetric particles have an influence on various observables we can measure. However this effect is typically suppressed by the particle's mass and relevant couplings and hence is often minuscule. Nevertheless, even minuscule effects can be detected by extremely precise measurements. One such measurement of importance is the anomalous magnetic moment of the muon, which is the contribution to the classical magnetic moment due to quantum fluctuations. There is at present a statistically significant, $3.3 \sigma$, discrepancy between the experimental value and that predicted by the standard model. This discrepancy may be one of the first indications of a supersymmetric effect. Assuming that the discrepancy is due to quantum fluctuations of supersymmetric particles implies an important constraint on supersymmetric models. If the mass of the super-partners is very large then the supersymmetric contribution to the anomalous magnetic moment of the muon is negligible. The supersymmetric contribution only makes up the required difference if some of the super-partners are light enough. This is a crucial constraint affecting the detectability of supersymmetry.

\subsection{Bayesian inference}

Supersymmetric models may be very compelling mathematical constructions, but studying them quickly raises the question: Is supersymmetry a symmetry of Nature? In the spirit of physics the answer to this question is obtained by contrasting supersymmetric theories with experiments. This is done by comparing various predictions of a selected supersymmetric model to experimental data of choice. However, predictions for the outcome of various experiments in the framework of a given supersymmetric model are not unique. They depend on theoretical assumptions about supersymmetry breaking and, within a given scenario, on the free parameters of the Lagrangian defining the model. This creates a serious obstacle to us learning if and how supersymmetry is realised in the physical Universe. While under the circumstances a straight yes or no answer can not be obtained to our original question, we can still answer various questions that provide vital information on supersymmetry. One of these questions is: Can a given supersymmetric model accommodate our experimental data at all? Another question that we can answer is: What are the values of the supersymmetric parameters most preferred by experiment?

We can answer these questions in a completely rigorous manner using statistics (Baer \& Balázs, 2003). The theoretical hypothesis that a certain supersymmetric model exists can be quantitatively tested by statistical methods such as Bayesian inference. Within this framework the answers to the above questions come in forms of likelihood maps over the parameter space of the supersymmetric model.

Such a likelihood map defined over the theoretical parameter space quantifying the supersymmetric hypothesis is $\mathcal{L}(D \mid P)$. It gives the probability density of obtaining a set of experimental outcome $D$ conditional on setting the theoretical parameters to values $P$. For various statistically uncorrelated measurements, this likelihood can be calculated as the product of likelihoods corresponding to the individual observations. In the case of normally 
distributed uncertainties, we have:

$$
\mathcal{L}(D \mid P)=\prod_{i=1}^{M} \frac{1}{\sqrt{2 \pi} \sigma_{i}} \exp \left(-\left(\frac{d_{i}-t_{i}(P)}{\sqrt{2} \sigma_{i}}\right)^{2}\right) .
$$

Here $d_{i}$ represent experimental central values of measurements and $t_{i}(P)$ are theoretical predictions that depend on the parameter values for the given experiments. For each observations the statistical and systematic experimental and theoretical uncertainties are quadratically combined into a single uncertainty $\sigma_{i}$. The number of observations is assumed to be $M$. For measurements that provide exclusion limits, such as searches with negative results, the likelihood is typically given by an error function instead of the above Gaussian form.

The likelihood map constructed over the whole parameter space quantitatively answers the question of whether the supersymmetric model accommodates the experimental data. A low likelihood function over the whole parameter space clearly indicates that the theory is in conflict with the considered experiments. If the value of the likelihood function is sufficiently high somewhere in the parameter space that means the parameter region can accommodate the given supersymmetric model.

While the likelihood function tells us the probability of the experimental outcome for a given set of parameters (under the supersymmetric hypothesis) it does not necessarily tell us the values of the supersymmetric parameters preferred by the data. This is because the latter is given by the conditional probability $\mathcal{P}(P \mid D)$ which, according to probability theory, is related to $\mathcal{L}(D \mid P)$ as

$$
\mathcal{P}(P \mid D) \mathcal{P}(D)=\mathcal{L}(D \mid P) \mathcal{P}(P) .
$$

The above relation is Bayes' theorem and it tells us that the joint probability of both $D$ and $P$ happening simultaneously can be expressed in two equivalent ways involving the probabilities $\mathcal{P}(D)$ and $\mathcal{P}(P)$ describing that $D$ and $P$ happens separately. Armed with this understanding we can calculate $\mathcal{P}(P \mid D)$ as the function of $\mathcal{L}(D \mid P)$ and give an answer to our second question regarding the preferred values of the parameters.

When aiming to infer parameter values from data by calculating $\mathcal{P}(P \mid D)$, we typically view the probability density $\mathcal{P}(P)$ as prior information on the parameters and refer to $\mathcal{P}(P)$ as the prior. In the same spirit the probability density $\mathcal{P}(P \mid D)$ itself is usually called the posterior, describing the inferred values of the parameters after information on the data was folded in. But how does $\mathcal{P}(P \mid D)$ "describe" the preferred value of a given parameter? The answer is clear when we get the rest of the parameters out of the way by integrating over them

$$
\mathcal{P}\left(p_{i} \mid D\right)=\int \mathcal{P}(P \mid D) \prod_{i \neq n=1}^{N} d p_{n} .
$$

In technical terms this is called the marginalization of the posterior to a given parameter $p_{i}$, and $N$ is the number of parameters quantifying the hypothesis. The function $\mathcal{P}\left(p_{i} \mid D\right)$, when interpreted as the function of a single variable $p_{i}$, gives the probability distribution of the parameter. The posterior can also be marginalized to two parameters

$$
\mathcal{P}\left(p_{i}, p_{j} \mid D\right)=\int \mathcal{P}(P \mid D) \prod_{i, j \neq n=1}^{N} d p_{n} .
$$


Since $\mathcal{P}\left(p_{i} \mid D\right)$ is the probability density of the parameter $p_{i}$, integrating it over the full allowed region of the parameter gives unity

$$
\int \mathcal{P}(P \mid D) \prod_{n=1}^{N} d p_{n}=1,
$$

expressing the fact that the value of the parameter should reside somewhere in its allowed region. This condition can be used to eliminate $\mathcal{P}(D)$ from the calculation of marginalized posterior distributions.

Once the posterior distribution is known we can determine credible intervals for each of the parameters. We define a credibility region $\mathcal{R}_{x}$ for a parameter $p_{i}$ by the minimal sized parameter region supporting $x$ percent of the total probability:

$$
x=\int_{\mathcal{R}_{x}} \mathcal{P}\left(p_{i} \mid D\right) d p_{i}
$$

In plain terms, a 68 percent credibility interval is the minimal parameter region that contains 68 percent of the area under the posterior distribution. This region gives the location of the parameter at 1- $\sigma$ certainty. Combined credibility regions over multi-dimensions of the parameter space can be similarly defined as the minimal region satisfying

$$
x=\int_{\mathcal{R}_{x}} \mathcal{P}\left(p_{i}, p_{j} \mid D\right) d p_{i} d p_{j} .
$$

The likelihood function can also be projected to one of its variables by forming profile distributions. These are constructed by finding the maximum likelihood hypersurface

$$
\mathcal{L}_{\max }\left(D \mid p_{i}\right)=\max _{p_{1}, \ldots, p_{i-1}, p_{i+1}, \ldots, p_{n}}(\mathcal{L}(D \mid P)) .
$$

and projecting this to one (or more) of the parameters (or to a function of the parameters). These profile likelihoods highlight the model regions where the likelihood is highest.

The numerical construction of a comprehensive likelihood map over a supersymmetric parameter space is required for the evaluation of the marginalized posteriors. This can be a challenging task and its essence is the satisfactory sampling of the likelihood function. The most efficient sampling methods focus on the parameter regions where the likelihood function is the highest. One of the simplest of these methods is the construction of a Markov chain. A Markov chain samples the parameter space in proportion with the value of the likelihood function. High likelihood regions are sampled proportionally higher using the Metropolis-Hastings algorithm. The chain is truncated when some convergence criterion is met. The power spectrum of the chain can be used to show when it is approaching the behaviour of a purely random walk, after which the sample is not gleaning much new information. Parameter regions with several local maxima typically require multiple chains to achieve the most faithful sampling.

In the Bayesian approach, in order to make statistical inferences about the parameter values we must include their prior probability $\mathcal{P}(P)$. Different choices of prior can lead to vastly different inferences. If no prior information is available about a given parameter the choice of a uniform prior appears to be justified. This choice is not trivial however. Should the prior, representing no a priori information, be uniform in the parameter itself or in some function 
of the parameters? The answer to this question is not obvious. Present practice in the field of supersymmetric phenomenology prefers two types of priors: uniform in the parameter itself or in the logarithm of the parameter. The latter reflects the lack of prior information on the order of magnitude of the parameter.

\section{Likelihood analysis of the next-to-minimal supergravity model}

In this section we present our findings for the viability of the NmSuGra model. Due to the complexity of the calculations and the number of observables involved, our numerical program comprised of a number of different codes compiled together, communicating via a sampler implementing the Metropolis algorithm. This code is tasked with selecting points in the parameter space and calculating the likelihood of the results collected. Each chosen set of input parameters is passed to the spectrum generator, NMSPEC (Ellwanger \& Hugonie, 2007), which produces the input required for the other codes to calculate their respective observables. These calculations, including the relic density, the anomalous magnetic moment of the muon $\left(\Delta a_{\mu}\right)$, and various quantities related to b-physics, are performed by MicrOMEGAS and other codes in NMSSMTools (Belanger et al., 2006).

For the free parameters of the NmSuGra model, we scan over the following ranges: $M_{0}<5$ $\mathrm{TeV}, M_{1 / 2}<2 \mathrm{TeV},-3 \mathrm{TeV}<A_{0}<5 \mathrm{TeV}, \tan \beta<60,10^{-5}<\lambda<0.6$. The positive choice of $\lambda$ ensures that the sign of the superpotential $\mu$ parameter is positive as this is strongly favoured by certain measurements including $\Delta a_{\mu}$ and the branching ratio $b \rightarrow s \gamma$. Seperate scans were performed using linear priors and logarithmic priors in order to gauge the dependence of the results obtained on the prior chosen. As the features of these plots are similar, we display only the linear priors, and simply explain the consequences of choosing a logarithmic prior.

We begin by plotting the profile likelihood for the input parameters of NmSuGra in Figure 1. Our first observation is simply that the magnitudes of the dimensionful parameters $\left(M_{0}, M_{1 / 2}\right.$, and $A_{0}$ ) appear to be constrained strongly by experiment. This is due largely to the anomalous magnetic moment of the muon, $\delta a_{\mu}$ (Balázs \& Carter, 2008). In general, as $M_{0}$ or $M_{1 / 2}$ increase, the masses of super-particles increase, and supersymmetric contributions to $\delta a_{\mu}$ decrease. Thus higher super particle masses incur a penalty to their likelihood up to a limit of $3.3 \sigma$ corresponding to $\Delta a_{\mu}=0$. In general, the combination of $\Delta a_{\mu}$ and $\Omega h^{2}$ defines many of the general features of the likelihood distribution. The likelihood is highest at relatively low values of $M_{0}$ and $M_{1 / 2}$ where light super particle masses help satisfy both constraints.

Figure 2 shows the marginalised posterior probability of the input parameters. The contrast with Figure 1 is considerable, particularly for $M_{0}$ and $\tan \beta$. This is caused by the so-called volume effect. The marginalised posterior represents the cumulative probability corresponding to each input value because of the integration in equation (12). As a result, a low average likelihood can be compensated for by simple spatial expanse. Thus, despite the approximately $3 \sigma$ penalty imposed on the likelihood by $\delta a_{\mu}$, high $M_{0}$ values may still be reasonable. The large volume elevating the posterior for high values of $M_{0}$, and contributing to the sharp peak at $\tan \beta \approx 50$ is called the focus point, shown clearly in Figure 3 . Conversely, low values of $\tan \beta$ severely constrict what regions of parameter space are permissible, so the posterior is low in spite of the high likelihood of the viable regions. Figure 2 does not imply any upper bounds on $M_{0}$, unlike $M_{1 / 2}$ and $A_{0}$, and unlike what the profile likelihoods suggest. Unfortunately, as $M_{0}$ directly influences the masses of sparticles, this may decrease the chances of colliders being able to produce them. 

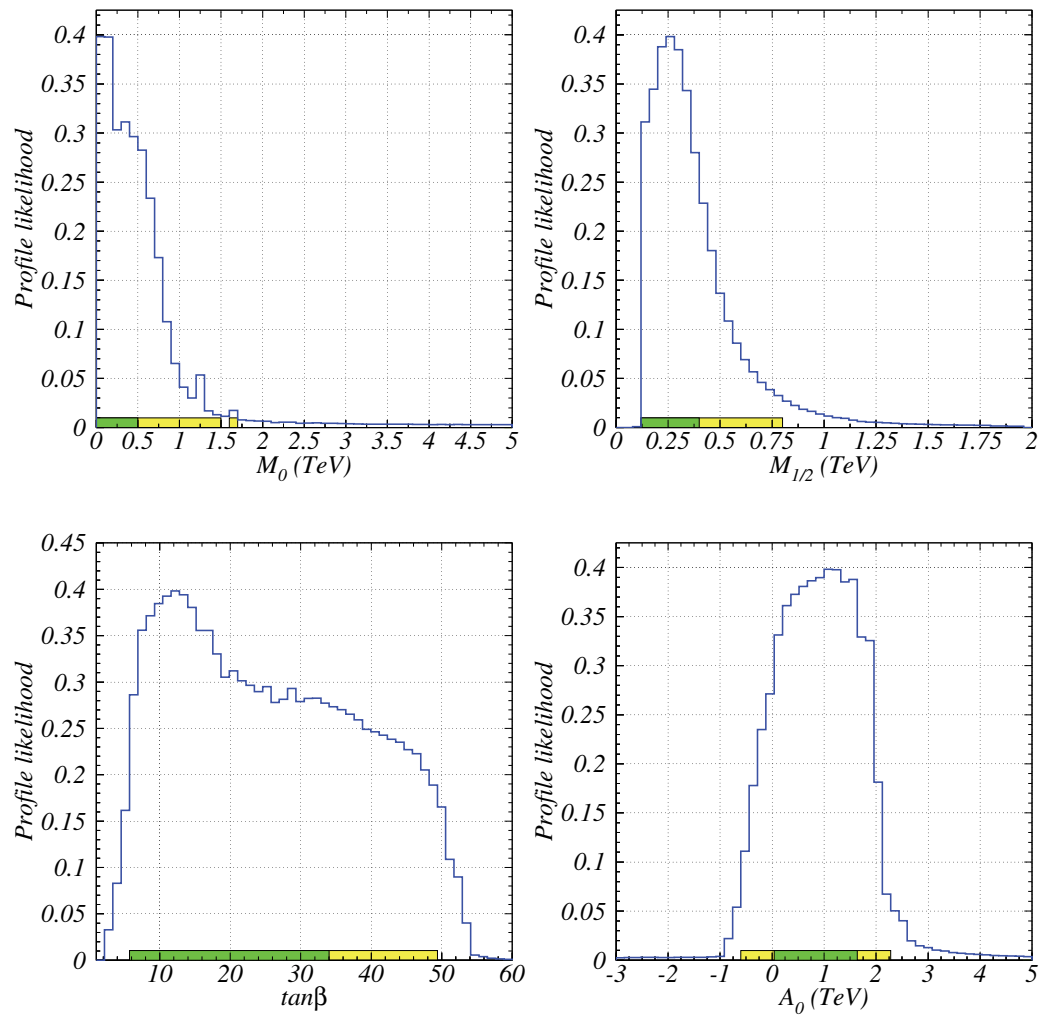

Fig. 1. Profile likelihoods of NmSuGra input parameters.

Naturally, we must keep in mind that these results are contingent on our choice of prior, and in changing this we may have to reconsider what inferences we draw. Logarithmic priors are agnostic to scale, making all orders of magnitude within the scan limits equally likely. Consequently, assuming logarithmic priors instead of linear can mitigate the probability enhancement that the larger volume at high parameter values entails. Certainly, these priors alleviate the potentially problematic high posterior at high $M_{0}$.

Our results in Figure 3 are useful for determining the properties of dark matter that we may anticipate for this model. Considering the top-left panel of Figure 3, different annihilation mechanisms can be identified from the structure of the posterior in parameter space. Looking towards the left, when the common scalar mass $M_{0}$ is sufficiently low, some scalar particle (generally the stau) becomes lighter than the lightest neutralino, which is forbidden under our assumption of electrically neutral dark matter. However, as the mass difference between these particles decreases the rate of mutual co-annihilation swiftly increases. Hence there is a narrow band of viable parameter space close to the left hand edge. This edge shifts however with the variation of the remaining parameters, so the marginalised posterior in $M_{0}$ and $M_{1 / 2}$ becomes smeared. Higgs resonances, where the lightest neutralino resonance-annihilates to a Higgs boson, occur along thin hypersurfaces where any of the Higgs masses are twice the 

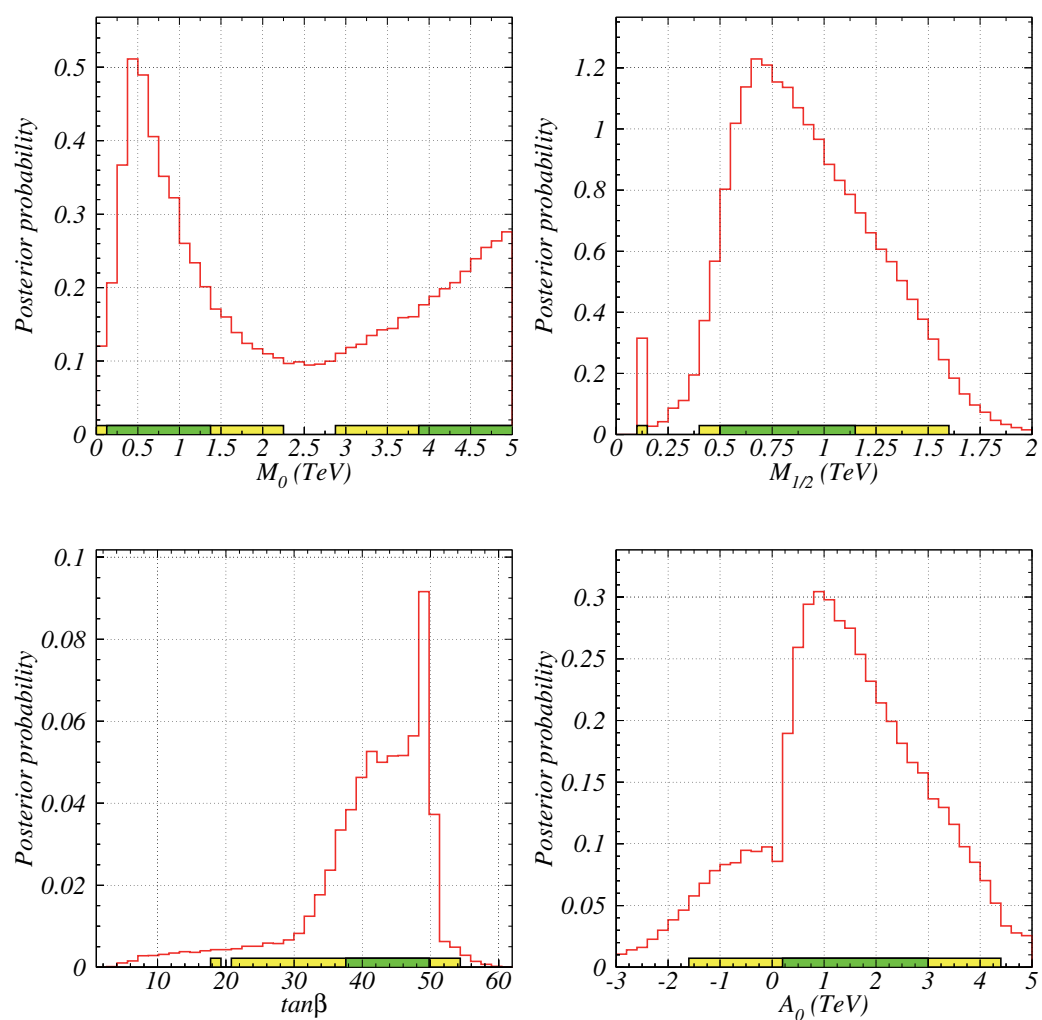

Fig. 2. Posterior probabilities with linear priors marginalised to individual input parameters.

neutralino mass. They can create high likelihood values in otherwise uninteresting regions. Their impact on the posterior can be considerable due to the volume of these Higgs funnels. Along right-hand side of the plot, towards higher $M_{0}$ values, we find the focus point region. Here the lightest neutralino is largely higgsino, and it co-annihilates with charginos and other neutralinos. The choice of logarithmic priors typically suppresses the posterior both in the Higgs resonance and focus point regions.

On the top left frame of Figure 3 the diagonal black curve shows the estimated reach of the LHC for $100 \mathrm{fb}^{-1}$ luminosity at $14 \mathrm{TeV}$ center of mass proton-proton collisions. It is evident that with $100 \mathrm{fb}^{-1}$ the LHC will be able to cover the stau-neutralino co-annihilation and part of the Higgs resonance corridors. But the LHC will stop short of fully exploring the high mass Higgs resonance annihilation region and the focus point at high $M_{0}$. To put this reach into context: LHC experimentalists celebrated the collection of data corresponding to the first $\mathrm{fb}^{-1}$ on June 16 of 2011. This luminosity was collected at half of the design energy, $7 \mathrm{TeV}$ center of mass (3.5 TeV proton beams). Presently $165 \mathrm{pb}^{-1}$ of this data is analysed in the context of the most constrained supersymmetric models (ATLAS collaboration, 2011). This data enables the LHC to reach up to about $M_{1 / 2}=450 \mathrm{GeV}$ at $M_{0}=100 \mathrm{GeV}$ and to about $M_{1 / 2}=240 \mathrm{GeV}$ 

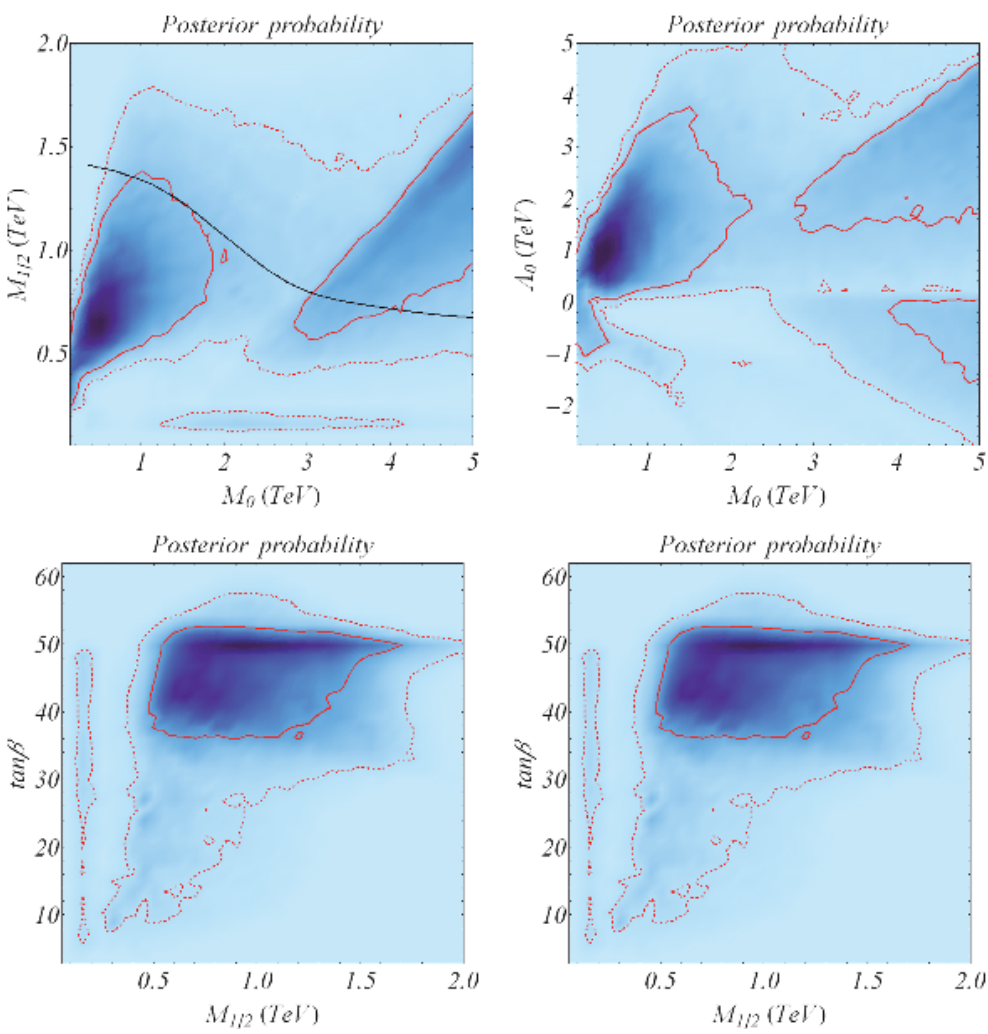

Fig. 3. Posterior probabilities with linear priors marginalised to pairs of input parameters. Various mechanisms for the annihilation of dark matter can be identified from the structure of the posterior distributions. On the top left frame the diagonal black curve shows the estimated reach of the LHC for $100 \mathrm{fb}^{-1}$ luminosity.

at $M_{0}=1000 \mathrm{GeV}$. As the top left frame of Figure 3 shows the LHC just started to probe the $68 \%$ confidence level region of the NmSuGra model.

Figures 4 and 5 show, respectively, the profile likelihoods and posterior probabilities of certain interesting super-partner masses. Again, the volume effect skews the posterior probability away from the profile likelihood toward higher values of $M_{0}$ and $M_{1 / 2}$. Although the likelihood can be high at low mass values, these occur only in a very confined region of parameter space. The volume effect is particularly noticeable in the stau mass which closely mimics the behaviour of $M_{0}$. Indeed, as the lightest stau is responsible for neutralino annihilation at low $M_{0}$ values, and largely responsible for the value of $\delta a_{\mu}$, the stau mass accounts to a large extent for the likelihood distribution of $M_{0}$. The gluino and stop masses are those of most relevance to detection at colliders, so it is promising that their masses are unlikely to be arbitrarily high (in this model at least). The mass of the lighest neutralino is less on average than the others in part simply because it must be the lightest supersymmetric particle. High neutralino masses generally incur penalties from $\delta a_{\mu}$. 

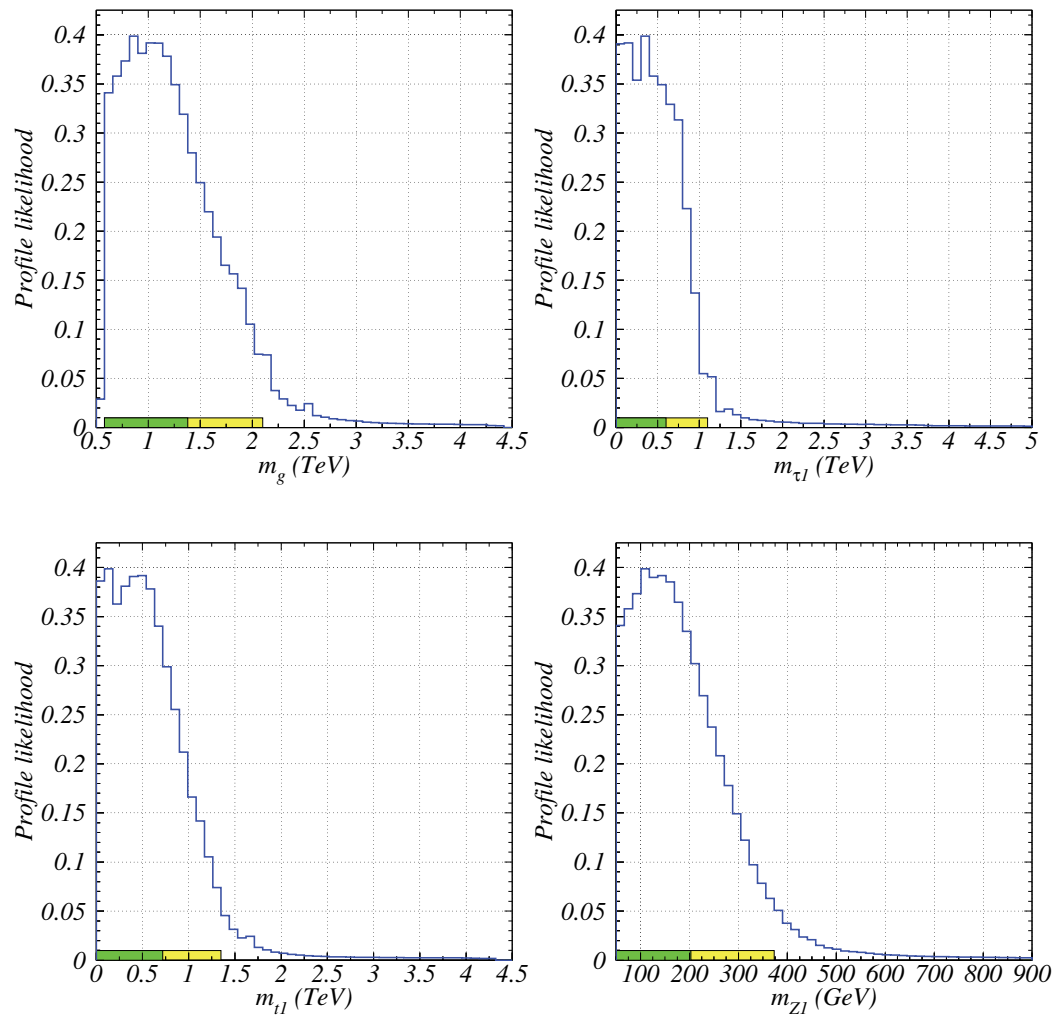

Fig. 4. Profile likelihood distributions for the masses of certain supersymmetric particles, the gluino (top-left), lightest stau (top-right), lightest stop (bottom-left), and lightest neutralino (bottom-right).

\subsection{Detection of supersymmetry}

The supersymmetric particle that is by far the most likely to be produced at a hadron collider will be one of the lightest strongly interacting super-particles, either the lightest squark (a stop in mSuGra) or the gluino. This particle will then decay in a chain that (assuming R-parity) must contain the lightest neutralino, and will hopefully lead to a discernable signal. Thus, to estimate the capability of a hadron collider to detect supersymmetry, we use the mass of the lightest stop and the gluino. Due to the similarity between the two models, we may apply results already obtained for the MSSM (Baer et al., 2003), assuming an eventual luminosity of $100 \mathrm{fb}^{-1}$.

The masses of the stop and the gluino weakly depend on $A_{0}$ and $\tan \beta$, so their contours may be plotted to a good approximation as lines in the $M_{0}-M_{1 / 2}$ plane. As shown in the first frame of Figure 3, this contour appears to enclose a large proportion of viable parameter space, which is good news for detection prospects.

It should be clear, of course, that particle mass is a fairly crude indication of the detectability of any given point in parameter space. The production of the gluino and squarks in a 

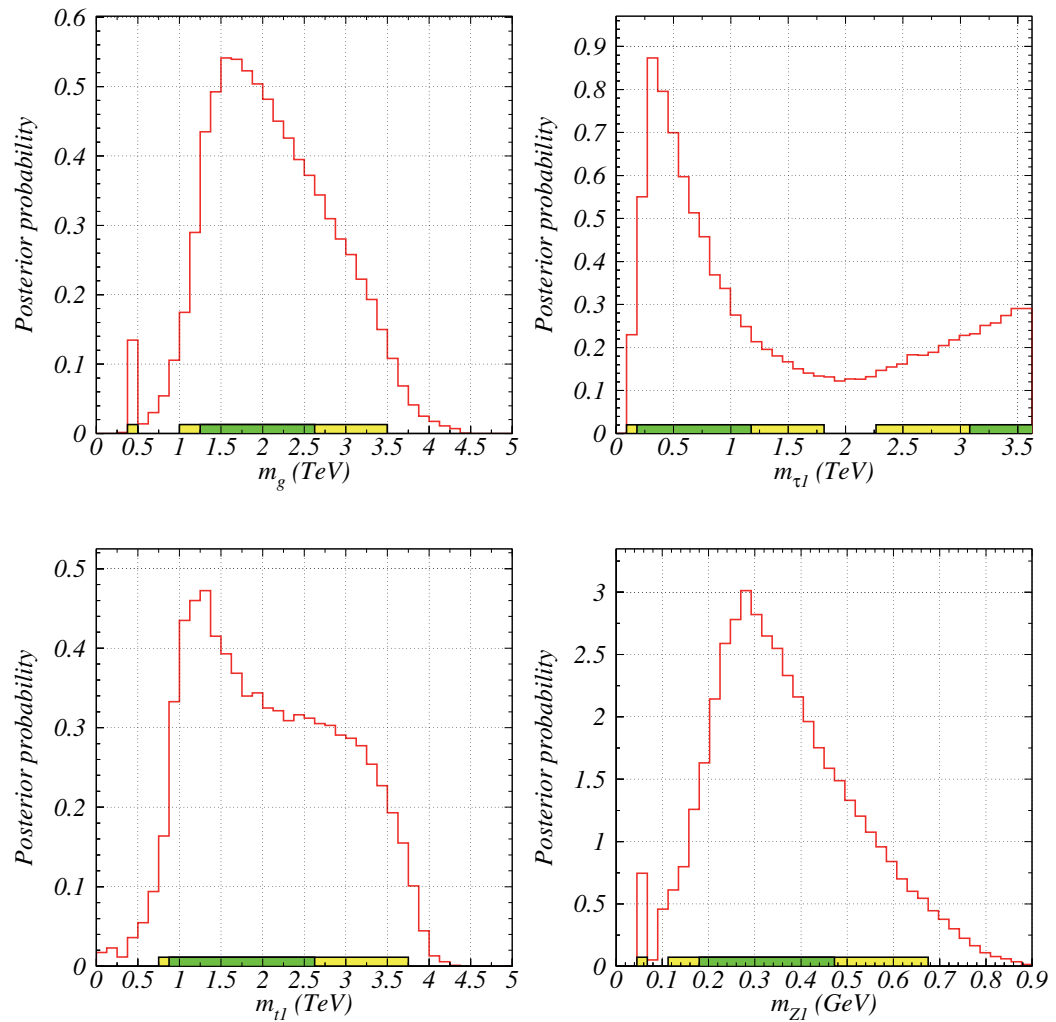

Fig. 5. Posterior probabilities with linear priors marginalised to selected super-particle masses.

hadron collider will be determined by the kinematics, so mass is obviously crucial. However, the significance of the signal (whether an exotic signal can be in fact be verified), depends sensitively on the details of the spectrum, and may be much more difficult to achieve than mere production. However, a full simulation of collider events and detection is a highly complex task, and unfeasible to do for every point in parameter space, so some simplification must be used.

In Figure 6 we show the potential for the direct detection of neutralinos from existing and upcoming experiments. Current exclusion limits are not extremely potent. However, large amounts of viable parameter space seem to be on the cusp of detectability. These points reside mainly in the focus point region. Rather fortuitously future direct detection experiments are able to cover much of the parameter space that will not be accessible to colliders. Based on our assumptions, the bulk of viable parameter space will be covered by either imminent collider or direct detection experiments. We may attempt to estimate the actual chances by quantifying the probability contained within the contours compared with the total amount residing in all parameter space. For the collider reach, we must integrate the posterior probability over all regions in parameter space satisfying either the stop or the gluino mass limit. Doing this 


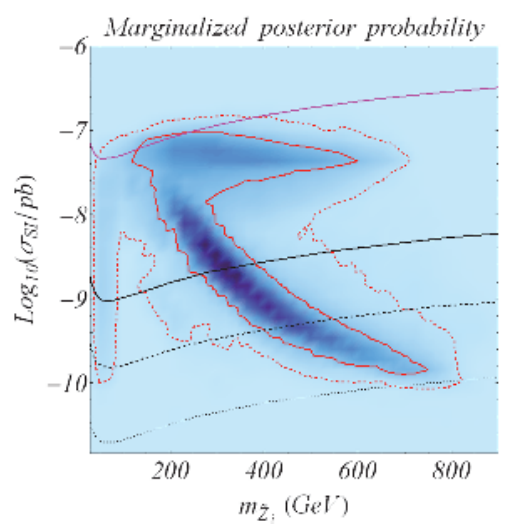

Fig. 6. Marginalised posterior probability in terms of the mass of the neutralino and the spin-independent contribution to the neutralino-neucleon cross-section. The present experimental limit is shown as a solid magenta line. The other limits, in order of increasing strength, represent the projected $25 \mathrm{~kg}, 100 \mathrm{~kg}$, and $1000 \mathrm{~kg}$ CDMS reach (Ahmed et al., 2009; Akerib et al., 2006).

calculation with linear priors, we obtain a chance of $57 \%$ that one of these super-particles will be produced at the large hadron collider (assuming, naturally, that our chosen theory is correct). Also encouragingly, with logarithmic priors our chances increase substantially due to the severe disfavour the focus point receives. Including points accessible to 1 ton CDMS, this estimate becomes $99.7 \%$, all but guaranteeing the eventual discovery of this model if it exists (Balázs \& Carter, 2010).

We close our discussion of supersymmetry detection with remarks on gravitational waves. This is because cosmological first order phase transitions may leave their imprint on gravitational waves in the early Universe, and the existence of supersymmetry may imply various such first order phase transitions. The breaking of supersymmetry, for example, may involve more than one metastable vacua. Relaxing via first order phase transitions these vacua may generate a background of gravitational waves. These gravitational waves, in turn, provide an insight into supersymmetry breaking. Supergravity motivated models, such as $\mathrm{NmSuGra}$, are based on a unification of all forces near $10^{16} \mathrm{GeV}$. Similarly to supersymmetry breaking the breaking of the unified gauge group may be accompanied by gravitational waves. If detected, these gravitational waves can support unification. While supersymmetry provides a dynamical explanation for the spontaneous breaking of electroweak symmetry it may similarly explain the matter-antimatter asymmetry of the Universe via electroweak baryogenesis Balazs et al. (2005). The latter requires the electroweak phase transition to be strongly first order. A strongly first order phase transition generates gravitational waves, the observation of which may shed further light on the mechanism of electroweak baryogenesis and the role of supersymmetry therein.

\section{Conclusion}

According to our analysis, the prospects for the detection of some supersymmetric particle are quite favourable, at least insofar as supergravity models are concerned. Our results show that the bulk of viable parameter space lies within the potential eventual reach of the LHC 
or direct detection experiments even for linear priors, and for logarithmic priors the odds are significantly better. The chances for finding the Higgs boson (again assuming something like supergravity is correct) are similarly promising.

As anticipated, the phenomenology we have observed for the constrained next-to-minimal supersymmetric standard model is not markedly different to what has been obtained for the constrained MSSM. In NmSuGra there are extended neutralino and Higgs sectors. This implies a potential singlet component for the lightest neutralino and Higgs, which could complicate detection attempts. We determine, however, that the presence of these novel features is very limited in the parameter space we explore.

As experimental limits and precision continue to improve, viable regions in parameter space for candidate models will become further constrained, and our ability to discriminate between them should increase. The detection of new particles would be particularly helpful, and between the Large Hadron Collider and dark matter detection experiments the chances for this seem quite reasonable.

\section{References}

Abazajian, K. N. et al. (2009). The Seventh Data Release of the Sloan Digital Sky Survey, Astrophys. J. Suppl. 182: 543-558.

Ahmed, Z. et al. (2009). Search for Weakly Interacting Massive Particles with the First Five-Tower Data from the Cryogenic Dark Matter Search at the Soudan Underground Laboratory, Phys. Rev. Lett. 102: 011301.

Akerib, D. S. et al. (2006). The SuperCDMS proposal for dark matter detection, Nucl. Instrum. Meth. A559: 411-413.

Amaldi, U., de Boer, W. \& Furstenau, H. (1991). Comparison of grand unified theories with electroweak and strong coupling constants measured at LEP, Phys. Lett. B260: 447-455.

Amsler, C. et al. (2008). Review of particle physics, Phys. Lett. B667: 1-1340.

ATLAS collaboration (2011). Search for squarks and gluinos using final states with jets and missing transverse momentum with the atlas detector in sqrts=7tev proton-proton collisions, http://cdsweb.cern.ch/record/1356194/.

Baer, H. \& Balázs, C. (2003). Chi**2 analysis of the minimal supergravity model including WMAP, $\mathrm{g}(\mathrm{mu})-2$ and $\mathrm{b}$ to s gamma constraints, JCAP 0305: 006.

Baer, H., Balázs, C., Belyaev, A., Krupovnickas, T. \& Tata, X. (2003). Updated reach of the CERN LHC and constraints from relic density, $b \rightarrow s$ gamma and $a(m u)$ in the mSUGRA model, JHEP 06: 054.

Baer, H. \& Tata, X. (2006). Weak scale supersymmetry: From superfields to scattering events.

Balazs, C., Carena, M. S., Menon, A., Morrissey, D. \& Wagner, C. (2005). The Supersymmetric origin of matter, Phys.Rev. D71: 075002.

Balázs, C. \& Carter, D. (2008). Discovery potential of the next-to-minimal supergravity motivated model, Phys.Rev. D78: 055001.

Balázs, C. \& Carter, D. (2010). Likelihood analysis of the next-to-minimal supergravity motivated model, JHEP 03: 016.

Belanger, G., Boudjema, F., Pukhov, A. \& Semenov, A. (2006). MicrOMEGAs: Version 1.3, Comput. Phys. Commun. 174: 577-604. 
Chamseddine, A. H., Arnowitt, R. L. \& Nath, P. (1982). Locally Supersymmetric Grand Unification, Phys.Rev.Lett. 49: 970.

Clowe, D. et al. (2006). A direct empirical proof of the existence of dark matter, Astrophys. J. 648: L109-L113.

Coleman, S. R. \& Mandula, J. (1967). All Possible Symmetries of the S Matrix, Phys.Rev. 159: 1251-1256.

Dimopoulos, S. \& Georgi, H. (1981). Softly Broken Supersymmetry and SU(5), Nucl.Phys. B193: 150.

Ellwanger, U. \& Hugonie, C. (2007). NMSPEC: A Fortran code for the sparticle and Higgs masses in the NMSSM with GUT scale boundary conditions, Comput. Phys. Commun. 177: 399-407.

Fayet, P. (1975). Supergauge Invariant Extension of the Higgs Mechanism and a Model for the electron and Its Neutrino, Nucl. Phys. B90: 104-124.

Goldberg, H. (1983). Constraint on the Photino Mass from Cosmology, Phys.Rev.Lett. 50: 1419.

Haag, R., Lopuszanski, J. T. \& Sohnius, M. (1975). All Possible Generators of Supersymmetries of the S Matrix, Nucl.Phys. B88: 257.

Kneib, J. P., Ellis, R. S., Smail, I., Couch, W. J. \& Sharples, R. M. (1996). Hubble Space Telescope Observations of the Lensing Cluster Abell 2218, Astrophys. J. 471: 643.

Kolb, E. W. \& Turner, M. S. (1990). The Early universe, Front. Phys. 69: 1-547.

Komatsu, E. et al. (2011). Seven-Year Wilkinson Microwave Anisotropy Probe (WMAP) Observations: Cosmological Interpretation, Astrophys. J. Suppl. 192: 18.

Markevitch, M. et al. (2004). Direct constraints on the dark matter self-interaction cross-section from the merging galaxy cluster 1E0657-56, Astrophys. J. 606: 819-824.

Martin, S. P. \& Vaughn, M. T. (1994). Two loop renormalization group equations for soft supersymmetry breaking couplings, Phys.Rev. D50: 2282.

Pagels, H. \& Primack, J. R. (1982). Supersymmetry, Cosmology and New TeV Physics, Phys. Rev. Lett. 48: 223.

Rubin, V. C. \& Ford, W.Kent, J. (1970). Rotation of the Andromeda Nebula from a Spectroscopic Survey of Emission Regions, Astrophys.J. 159: 379-403.

Wess, J. \& Zumino, B. (1974). Supergauge Transformations in Four-Dimensions, Nucl. Phys. B70: 39-50. 


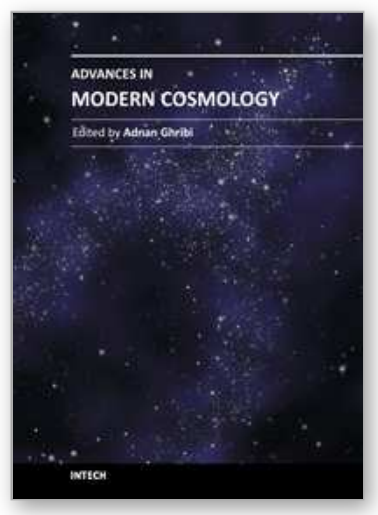

\author{
Advances in Modern Cosmology \\ Edited by Dr. Adnan Ghribi
}

ISBN 978-953-307-423-8

Hard cover, 198 pages

Publisher InTech

Published online 29, August, 2011

Published in print edition August, 2011

The twentieth century elevated our understanding of the Universe from its early stages to what it is today and what is to become of it. Cosmology is the weapon that utilizes all the scientific tools that we have created to feel less lost in the immensity of our Universe. The standard model is the theory that explains the best what we observe. Even with all the successes that this theory had, two main questions are still to be answered: What is the nature of dark matter and dark energy? This book attempts to understand these questions while giving some of the most promising advances in modern cosmology.

\title{
How to reference
}

In order to correctly reference this scholarly work, feel free to copy and paste the following:

Csaba Balázs and Daniel Carter (2011). Supersymmetric Dark Matter, Advances in Modern Cosmology, Dr. Adnan Ghribi (Ed.), ISBN: 978-953-307-423-8, InTech, Available from:

http://www.intechopen.com/books/advances-in-modern-cosmology/supersymmetric-dark-matter

\section{INTECH}

open science | open minds

\section{InTech Europe}

University Campus STeP Ri

Slavka Krautzeka 83/A

51000 Rijeka, Croatia

Phone: +385 (51) 770447

Fax: +385 (51) 686166

www.intechopen.com

\section{InTech China}

Unit 405, Office Block, Hotel Equatorial Shanghai

No.65, Yan An Road (West), Shanghai, 200040, China 中国上海市延安西路65号上海国际贵都大饭店办公楼 405 单元

Phone: +86-21-62489820

Fax: $+86-21-62489821$ 
(C) 2011 The Author(s). Licensee IntechOpen. This chapter is distributed under the terms of the Creative Commons Attribution-NonCommercialShareAlike-3.0 License, which permits use, distribution and reproduction for non-commercial purposes, provided the original is properly cited and derivative works building on this content are distributed under the same license. 